\title{
La cuádruple combinación de antihipertensivos a bajas dosis tendría un efecto aditivo beneficioso
}

\author{
The quadruple combination of antihypertensives at low doses could have a beneficial additive effect
}

\section{Objetivos}

Determinar la eficacia y la tolerancia de un tratamiento antihipertensivo combinado de cuatro drogas a un cuarto de la dosis habitual cada una, en pacientes con hipertensión, vírgenes de tratamiento.

\section{Diseño, lugar y pacientes}

Ensayo clínico aleatorizado, doble ciego, controlado con placebo y con diseño entrecruzado (crossover) realizado en cuatro centros comunitarios de atención primaria del oeste de Sidney, Australia. Participaron mayores de 18 años con una presión arterial sistólica (PAS) en el consultorio mayor de 140 y/o diastólica mayor de $90 \mathrm{mmHg}$ en dos mediciones durante días diferentes, o bien con una PAS ambulatoria mayor de 135 y/o una PAD ambulatoria mayor de $85 \mathrm{mmHg}$, vírgenes de tratamiento antihipertensivo.

Intervención y medición de los resultados principales Los pacientes fueron aleatorizados en proporción uno a uno a través de una secuencia computarizada a un esquema de cuatro semanas de una cuádruple píldora -una única píldora encapsu-
Chow C y col. Lancet 2017; 389:1035-42. lada conteniendo $37,5 \mathrm{mg}$ de irbesartan, $1,25 \mathrm{mg}$ de amlodipina, $6,25 \mathrm{mg}$ de hidroclorotiazida y $12,5 \mathrm{mg}$ de atenolol- seguido por una fase de lavado (wash-out) con placebo durante dos semanas y luego, por otras cuatro semanas de placebo; o bien a cuatro semanas de placebo inicial, seguidas por el mismo lapso de lavado y finalmente, cuatro semanas de tratamiento activo. Tanto los participantes como los investigadores del estudio estuvieron ciegos al orden de la asignación. El punto final primario fue la reducción de la PAS de $24 \mathrm{~h}$ a las cuatro semanas, evaluada a través de un Monitoreo Ambulatorio de Presión Arterial (MAPA). El análisis se realizó por intención de tratar.

\section{Resultados principales}

De los 55 individuos inicialmente evaluados, 21 fueron considerados elegibles y aleatorizados. Un participante abandonó el estudio antes de iniciar el tratamiento y otros dos lo hicieron luego del primer periodo de tratamiento, quedando 18 con datos completos para el análisis.

Respecto del punto final primario, se observó una reducción significativa de la PAS en el grupo tratamiento activo en comparación con el grupo placebo (ver tabla 1).

Tabla 1. Comparación de la eficacia de una píldora con dosis bajas de cuatro antihipertensivos y el placebo.

\begin{tabular}{l|c|c|c|c|c|c} 
& \multicolumn{2}{|c|}{ Periodo de la intervención } & \multicolumn{2}{|c|}{ Periodo de placebo } & \multirow{2}{*}{$\begin{array}{c}\text { Diferencia } \\
\text { (IC 95\%) }\end{array}$} & p \\
\cline { 2 - 6 } & Basal & Final & Basal & Final & $18,7(14,3$ a 23,0$)$ & $<0,0001$ \\
\hline $\begin{array}{l}\text { PAS en 24 h } \\
\text { Media (DE) }\end{array}$ & $138,4(7,3)$ & $119,6(7,6)$ & $137,1(10,4)$ & $138,2(10,0)$ & & \\
\hline
\end{tabular}

PAS: presión arterial sistólica.

Conclusiones

Este estudio sugiere que los beneficios del tratamiento con un cuarto de dosis podrían ser aditivos a través de las distintas clases de antihipertensivos, lo que conferiría una reducción clínicamente relevante de la presión arterial. Se requiere una mayor investigación del concepto de la cuádruple píldora para determi-

\section{Comentario}

El adecuado control de la presión arterial (PA) es escaso a nivel mundial ${ }^{1}$. Entre los principales factores responsables se encuentran la escasa adherencia al tratamiento por parte de los pacientes y la inercia terapéutica por parte de los médicos.

La mayoría de los pacientes tiene prescripta una sola droga ${ }^{2}$, a pesar de que se ha demostrado que la combinación de antihipertensivos a bajas dosis es más efectiva que la monoterapia a altas dosis ${ }^{3}$. En este contexto, resulta atractivo un enfoque que utiliza varias drogas a dosis bajas en una única píldora, buscando maximizar la adherencia y la eficacia, y minimizando los efectos adversos. Si bien el estudio que acabamos de resumir tiene la ventaja de haber confirmado el diagnóstico de hipertensión por MAPA y de utilizar también este método -de mayor valor pronóstico que la PA de consultorio- para evaluar el punto final primario, dos cuestiones metodológicas atentan contra la validez de sus resultados. Por un lado, el criterio utilizado aquí para definir nar su efectividad en comparación con el tratamiento habitual y la tolerancia a largo plazo.

Fuente de financiamiento/conflicto de interés de los autores: $\mathrm{Na}$ tional Heart Foundation, Australia; University of Sydney; National Health and Medical Research Council of Australia. La autora declara no tener conflicto de intereses.

adecuado control de la PA por MAPA fue más laxo que el de los estándares actuales ${ }^{4}$, lo que implica que en realidad alcanzaron buen control de la PA por MAPA menos del $83 \%$ de los pacientes en tratamiento activo reportados en este estudio. Por otro lado, los autores minimizaron los efectos adversos de laboratorio, que fueron más frecuentes durante el tratamiento activo, sin aclarar si los dos pacientes que abandonaron el estudio lo hicieron luego de la fase del tratamiento con placebo o con tratamiento activo.

\section{Conclusiones de la comentadora}

Este estudio sugiere -con algunas limitaciones metodológicas- el beneficio del tratamiento activo vs. placebo en la reducción de la PA de las personas con hipertensión arterial, sin embargo no debemos olvidar que no responde una pregunta mucho más relevante: si el enfoque planteado con la píldora cuádruple es mejor al enfoque actual en términos de eficacia, eficiencia y tolerancia.

Jessica Barochiner [ Sección Hipertensión Arterial, Servicio de Clínica Médica, Hospital Italiano de Buenos Aires. jessica.barochiner@hospitalitaliano.org.ar ] Barochiner J. La cuádruple combinación de antihipertensivos a bajas dosis tendría un efecto aditivo beneficioso. Evid Act Pract Ambul 2017;20(4):97. Comentado de: Chow C y col. Quarter-dose quadruple combination therapy for initial treatment of hypertension: placebo-controlled, crossover, randomised trial and systematic review. Lancet. 2017; 389(10073):1035-1042

Referencia bibliográficas

1. Chow $\mathrm{C}$ y col. PURE (Prospective Urban Rural Epidemiology) Study investigators. Prevalence, awareness, treatment, and control of hypertension in rural and urban communities in high-, middle-, and low-income countries. JAMA. 2013 Sep 4;310(9):959-68.

2.Marin M y col. Registro Nacional de Hipertensión Arterial: Conocimiento, tratamiento y control de la hipertensión arterial. Estudio RENATA. Revista argentina de cardiología. 2012; 80(2):121-129.

3.Wald D y col. Combination therapy versus monotherapy in reducing blood pressure: meta-analysis on 11,000 participants from 42 trials. Am J Med. 2009 Mar; $122(3): 290-300$. 3.Wald D y col. Combination therapy versus monotherapy in reducing blood pressure: meta-analysis on 11,000 participants from 42 trials. Am J Med. 2009 Mar; $122(3): 290-300$.
4. O'Brien E y col. European Society of Hypertension Working Group on Blood Pressure Monitoring.. European Society of Hypertension position paper on ambulatory blood pressure monitoring. J Hypertens. 2013 Sep;31(9):1731-68. 